\title{
Energy distribution of precipitating electrons estimated from optical and cosmic noise absorption measurements
}

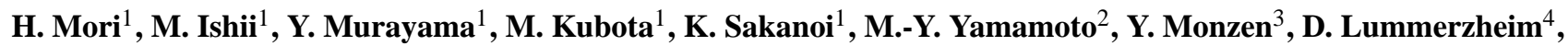 \\ and B. J. Watkins ${ }^{4}$ \\ ${ }^{1}$ Communications Research Laboratory, 4-2-1, Nukui-Kitamachi, Koganei, Tokyo, Japan \\ ${ }^{2}$ Kochi University of Technology, 185, Miyanokuchi, Tosayamada, Kochi, Japan \\ ${ }^{3}$ University of Electro-Communications, 1-5-1, Chofugaoka, Chofu, Tokyo, Japan \\ ${ }^{4}$ Geophysical Institute, University of Alaska Fairbanks, Fairbanks, Alaska 99775-7320, USA
}

Received: 19 August 2003 - Revised: 8 December 2003 - Accepted: 14 January 2004 - Published: 8 April 2004

\begin{abstract}
This study is a statistical analysis on energy distribution of precipitating electrons, based on CNA (cosmic noise absorption) data obtained from the 256-element imaging riometer in Poker Flat, Alaska $\left(65.11^{\circ} \mathrm{N}, 147.42^{\circ} \mathrm{W}\right)$, and optical data measured with an MSP (Meridian Scanning Photometer) over 79 days during the winter periods from 1996 to 1998 . On the assumption that energy distributions of precipitating electrons represent Maxwellian distributions, CNA is estimated based on the observation data of auroral 427.8-nm and 630.0-nm emissions, as well as the average atmospheric model, and compared with the actual observation data. Although the observation data have a broad distribution, they show systematically larger CNA than the model estimate. CNA determination using kappa or double Maxwellian distributions, instead of Maxwellian distributions, better explains the distribution of observed CNA data. Kappa distributions represent a typical energy distribution of electrons in the plasma sheet of the magnetosphere, the source region of precipitating electrons. Pure kappas are more likely during quiet times - and quiet times are more likely than active times. This result suggests that the energy distribution of precipitating electrons reflects the energy distribution of electrons in the plasma sheet.
\end{abstract}

Key words. Ionosphere (auroral ionosphere; particle precipitation; polar ionosphere)

\section{Introduction}

The high-energy precipitating electrons on the polar region during high geomagnetic activity play an important role for energetics and dynamics of ionospheric plasma, ionization, and neutral dynamics. Estimating the energy of the flux is

Correspondence to: $\mathrm{M}$. Ishii

(mishii@crl.go.jp) therefore an important issue in the study of the polar atmosphere.

Many studies have been conducted on the use of auroral optical measurements for estimating the energy of precipitating electrons (e.g. Rees, 1963; Rees and Luckey, 1974; Lummerzheim et al., 1990). As the aurora is a luminous phenomenon caused by electrons with energy lower than 10 $20 \mathrm{keV}$ in the E- and F-regions of the ionosphere, energy estimates using auroral emission are considered an effective way of measuring precipitating electrons in this energy range.

Another phenomenon associated with electron precipitation is CNA (cosmic noise absorption) (e.g. Holt and Omholt, 1962; Ansari, 1964; Johansen, 1965). CNA is mainly associated with increased electron density in the D-region of the ionosphere, caused by high-energy components of precipitating electrons at several tens of $\mathrm{keV}$. Therefore, comparing the optical and CNA observation data is expected to provide an energy estimate of precipitating electrons covering a broader energy range up to several tens of keV. Statistical analysis of electron energy spectra using data from NOAA/TIROS polar orbiting satellites (Codrescu et al., 1997) indicates that precipitation in the energy range above $30 \mathrm{keV}$ is common in the auroral zone, and enhances the electron density in the altitude range of $75-90 \mathrm{~km}$ by more than a factor of 2 . While Codrescu et al. (1997) describe the high energy precipitation with an additional Maxwellian distribution, Sharber et al. (1998) suggest that based on UARS/PEM observations during the November 1993 storm, a kappa distribution is more appropriate. Likewise, Frahm et al. (1997) (also see the comment by Callis, 2000, and the reply by Frahm et al., 2000) have found that a kappa distribution fits spectra over diffuse aurora. CNA observations are sensitive to these high energy tails of the auroral electrons, and with the analysis of riometer data in this paper we support the importance of the high energy precipitation. 

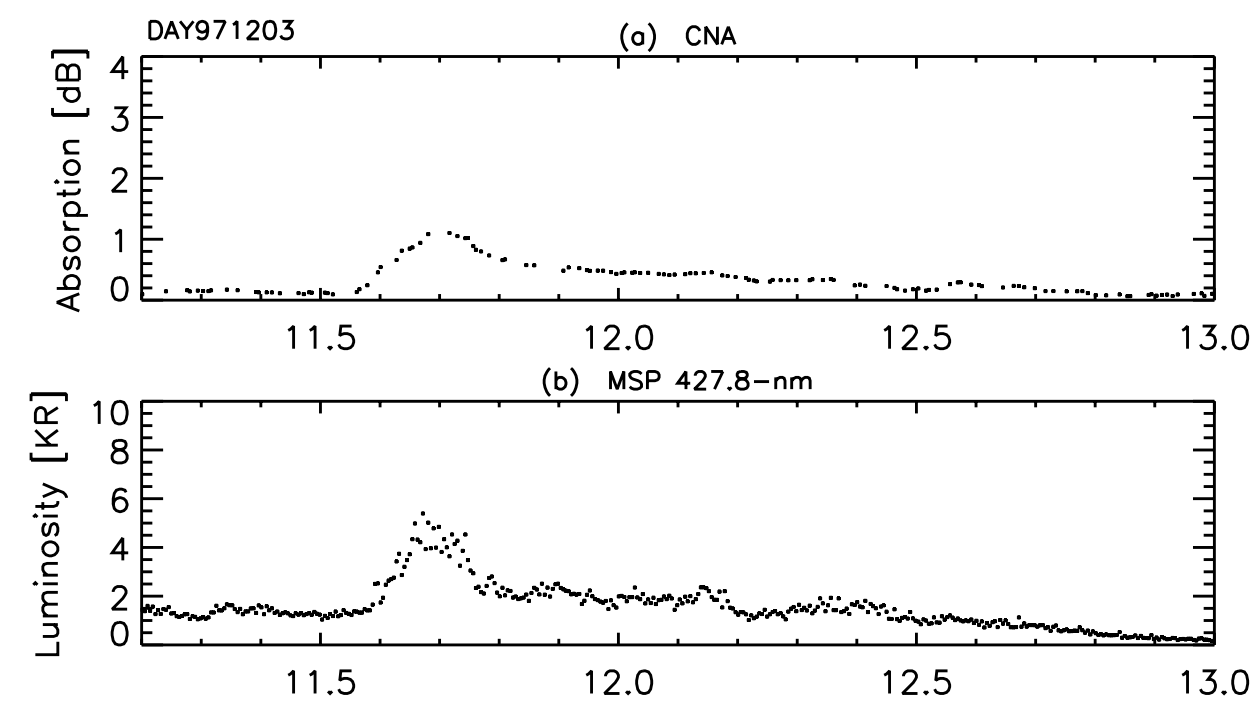

(c) MSP $630.0-\mathrm{nm}$
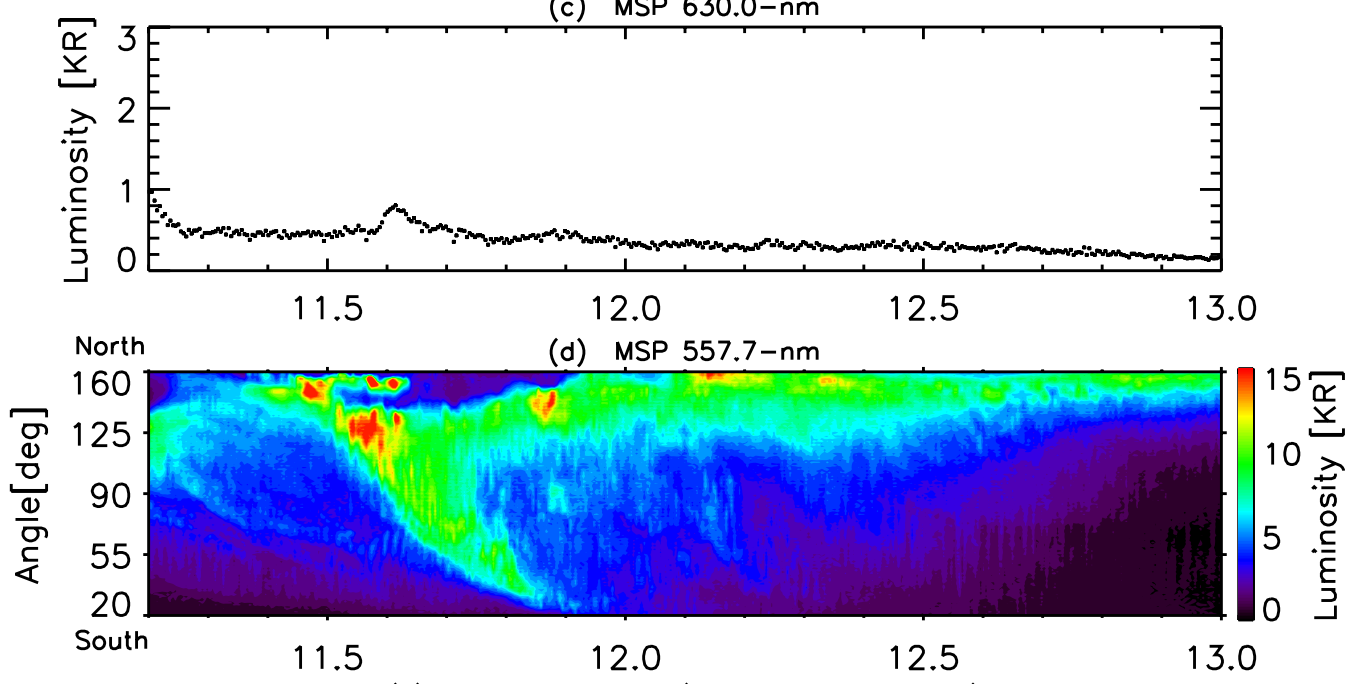

(e) MAG-X at PFRR (12510 nT subtracted)

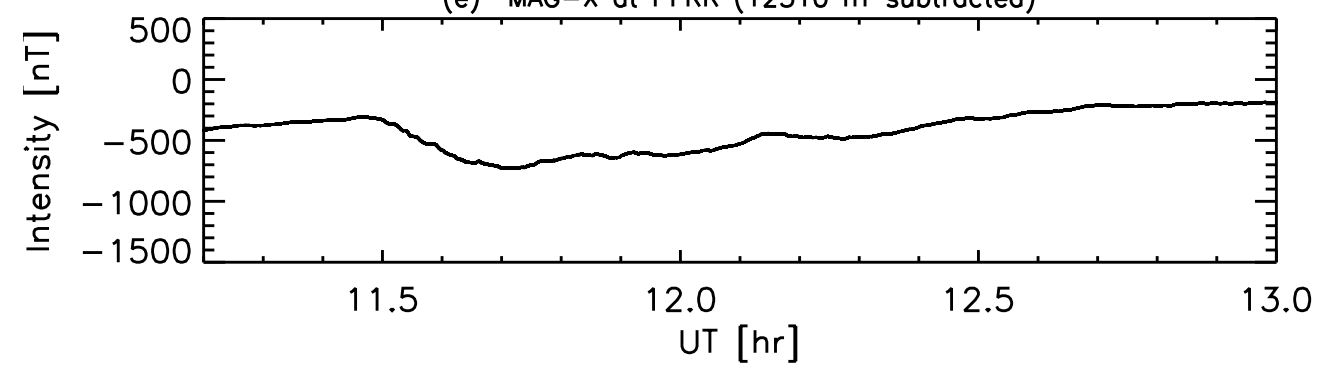

Fig. 1. Auroral activity observed in Poker Flat, Alaska, at 11:12 to 13:00 UT on 3 December 1997. (a) CNA in the direction of the geomagnetic zenith, measured by the imaging riometer. (b) 427.8-nm brightness in the direction of the geomagnetic zenith, measured by the MSP (Meridian Scanning Photometer). (c) 630.0-nm brightness in the direction of the geomagnetic zenith, measured by the MSP. (d) Keogram of the 557.7-nm brightness, from the MSP. (e) The geomagnetic field X (south-north) component at Poker Flat.

CNA is observed with a riometer (e.g. Little and Leinbach, 1959) which has a low spatial resolution because of the use of a wide-beam antenna. Yet, in the polar region, electron precipitation often occurs in spatially localized areas. Therefore, it is necessary to observe CNA with an appropriate spatial resolution. Detrick and Rosenberg (1990) developed an imaging riometer (IRIS, Imaging Riometer for Ionospheric
Studies) combining a 64-element antenna array and a Butler matrix for phase synthesis (Butler and Lowe, 1961) for observing CNA's spatial structure and its temporal variations.

The Communications Research Laboratory is conducting a joint middle and upper atmosphere research program with the Geophysical Institute, University of Alaska Fairbanks. As part of the activity, we have developed an imaging 
riometer using the IRIS technology with enhanced spatial resolution, featuring a 256-element antenna array, and installed it at Poker Flat, Alaska (geographic coordinates of $65.11^{\circ} \mathrm{N}, 147.42^{\circ} \mathrm{W}$, geomagnetic coordinates of $65.4^{\circ} \mathrm{N}$, $100.7^{\circ} \mathrm{W}$ ) to conduct observations starting in October 1995.

This paper presents a comparative analysis of CNA measurements obtained from this imaging riometer, and auroral spectroscopic emission data obtained from an MSP (Meridian Scanning Photometer) installed at the same location as the riometer, so as to examine energy distribution of precipitating electrons up to several tens of keV. First, auroral optical measurements are used to estimate the energy flux and the characteristic energy of precipitating electrons in a conventional method. The results are then combined with the MSIS-90 atmospheric model (Hedin, 1991) and average atmospheric parameters to estimate CNA, which is then compared with actual CNA measurements. The results of this statistical analysis, based on the 79-day data set, are used to estimate the energy distribution of precipitating electrons up to several tens of $\mathrm{keV}$. Section 2 describes the instrumentation and data processing, while Sect. 3 explains the method of estimating CNA based on auroral optical measurements. Section 4 details observation results, and Sect. 5 discusses the results. Section 6 is dedicated to a summary.

\section{Instrumentation and data processing}

The imaging riometer installed at Poker Flat features a 256-element crossed-dipole antenna, arranged in the $16 \times 16$ square array parallel to the geomagnetic latitude and longitude. Combined with the Butler matrix, it conducts observations in 208 effective beam directions within $70^{\circ}$ from zenith $\left(6^{\circ}\right.$ in two-way half-power width around the beam direction, $7^{\circ}$ in beam interval). The observing frequency is at $38.2 \mathrm{MHz}$, with the observation range of $400 \times 400 \mathrm{~km}$ at the altitude of $90 \mathrm{~km}$, and horizontal spatial resolution of approximately $11 \mathrm{~km}$ around the zenith. The phase synthesis output from the Butler matrix to 256 directions is detected with 16 receivers at every $1 / 16 \mathrm{~s}$, so that two-dimensional image observations can be conducted at the rate of each pattern per second. (Detailed specifications are shown in Murayama et al., 1997). CNA is given in $\mathrm{dB}$ as logarithmic value of the ratio of observed values and QDC (Quiet Day Curve), i.e. the monthly average of the receivers' output power.

As for auroral optical data, this paper used the observations from the MSP (Meridian Scanning tilting filter Photometer), which the University of Alaska has installed at Poker Flat. In this MSP, the central wavelength of auroral emission through a filter of 0.3 to $0.4 \mathrm{~nm}$ bandwidth, as well as the wavelength 0.5 to $1.0 \mathrm{~nm}$ offset from the central wavelength for observing the background continuum, are alternately scanned every $4 \mathrm{~s}$ along the geomagnetic meridian. The difference of the on-line and off-line brightness is used to obtain the true intensity. This analysis used values every $16 \mathrm{~s}$, after averaging the results of two consecutive

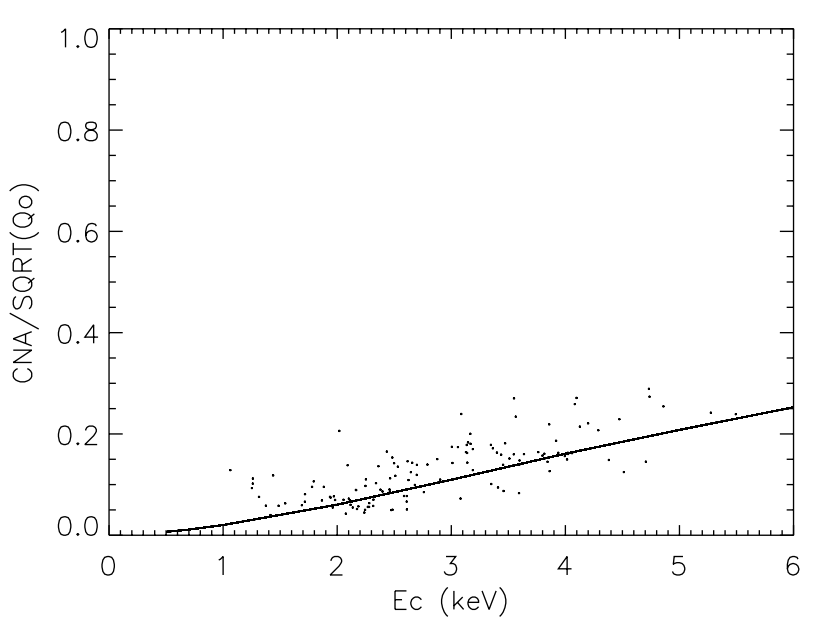

Fig. 2. Scatter plot of all CNA measurements normalized with the square root of the total energy flux $Q_{0}$ shown in Fig. 1 against the characteristic energy $E_{c} . E_{c}$ and $Q_{0}$ are obtained from the measurements of auroral 427.8-nm and 630.0-nm brightness. The solid line in this figure represents the theoretically estimated curve for $\mathrm{CNA} / \sqrt{Q_{0}}$ against $E_{c}$, as calculated with a Maxwellian distribution assumed as the energy distribution of precipitating electrons. In this case, the observed CNA values are generally distributed around the predicted curve.

scans. The field-of-view of an instantaneous observation is $1^{\circ}$ (Lummerzheim et al., 1990).

To monitor aurora activities generated at around Poker Flat, we referred to geomagnetic field measurements from a fluxgate magnetometer, installed at Poker Flat by the University of Alaska.

As described above, CNA and auroral emission measurements have different temporal and spatial resolutions. To ensure that the two sets of data can be compared under the same conditions, we adjusted the temporal resolution of the data according to the temporal resolution of the MSP data, the spatial resolution according to that of the imaging riometer beams.

Generally, auroral emission and CNA are both generated by energetic particles precipitating along magnetic field lines, but the stopping heights are different from each other. Observations in any off-zenith direction can only be related, if the altitude of the emission and absorption is known. Because we are measuring column-integrated quantities, we have no altitude information. Comparative analysis was therefore conducted based on data close to the geomagnetic zenith. For CNA, the output of four beams closest to the geomagnetic zenith was averaged to obtain the value for the direction of geomagnetic zenith. The value for auroral emissions was obtained through averaging the data over an angle corresponding to the beam interval of the imaging riometer around the direction of the geomagnetic zenith. The extinction and scattering of auroral intensity is adjusted with a factor for the clear sky condition in Poker Flat, as provided in Table 1; Lummerzheim et al. (1990). 


\section{Estimate of the energy of precipitating electrons and CNA using auroral spectroscopic emissions}

This section describes the conventional method of estimating precipitating electron energy using auroral spectroscopic emissions, and estimating CNA based on the obtained energy flux and characteristic energy.

First, the energy of precipitating electrons using auroral spectroscopic emissions is estimated as follows. Based on the differential-directional number flux for a downward isotropic Maxwellian energy spectrum of precipitating electrons $\mathrm{I}(\mathrm{E})$,

$I(E)=\frac{Q_{0}}{2 \pi E_{c}^{3}} E \exp \left(-\frac{E}{E_{c}}\right)$,

optical observation data are used to estimate parameters included in the distribution. $Q_{0}$ and $E_{c}$ are the total energy flux and the characteristic energy, respectively. The measurements of the $\mathrm{N}_{2}^{+} 1 \mathrm{~N}$ band $427.8-\mathrm{nm}$ and the OI line 630.0-nm were used as optical measurements (e.g. Rees and Luckey, 1974; Lummerzheim et al., 1990). $Q_{0}$ is obtained from the 427.8-nm intensity I [427.8] by the relation $Q_{0}\left(\mathrm{~mW} / \mathrm{m}^{2}\right)=\mathrm{I}[427.8] / 0.250(\mathrm{kR})$, and $E_{c}$ is estimated from the ratio of the $630.0-\mathrm{nm}$ intensity against the $427.8-\mathrm{nm}$ intensity, as shown in Fig. 7 by Lummerzheim et al. (1990).

The method of estimating CNA generated by precipitating electrons is based on a theoretical model. First, the energy distribution of precipitating electrons, obtained from the auroral brightness, and an appropriate atmospheric model are used to calculate the energy deposition rate of precipitating electrons at each altitude. In this paper, MSIS-90 is used as the atmospheric model, and the calculation is conducted according to the method given by Jackman et al. (1980). Dividing the energy deposition rate by the average energy loss of $35 \mathrm{eV}$ per ionizing collision (Valentine and Curran, 1958) will provide the ion pair production rate $q$ as the function of altitude $h$. Under the quasi-equilibrium condition, the ratio of $q$ and the effective recombination coefficient $\alpha_{\text {eff }}$ provides the electron density $N_{e}$, as shown in the following equation (e.g. Collis et al., 1984),

$N_{e}(h)=\sqrt{\frac{q(h)}{\alpha_{e f f}(h)}}$.

The electron density $N_{e}$ is used in the following height integration to obtain CNA:

$$
\begin{aligned}
& A(d B)=\int_{0}^{\infty} a(h) N_{e}(h) d h, \\
& a(h)=\frac{4.6 \times 10^{-5}}{\omega^{2}} v_{e n}(h) .
\end{aligned}
$$

Here, $A(\mathrm{~dB}), \omega$ and $v_{e n}(h)$ are the CNA, observation angular frequency, and electron-neutral collision frequency, respectively, while $a(h)$ is a function called specific absorption, defined as the amount of absorption (dB) by a single electron per cubic centimeter in a $1 \mathrm{~km}$ column (e.g. Parthasarathy and Berkey, 1965). In this paper, the values in Fig. 15 by Hargreaves and Devlin (1990) are used for the average effective recombination coefficient, $\alpha_{e f f}$, and the specific absorption, $a(h)$, in the auroral region. However, this specific absorption is for an observation frequency of $30 \mathrm{MHz}$, and is therefore multiplied with the adjustment coefficient $(30.0 / 38.2)^{2}$ according to our observation frequency of $38.2 \mathrm{MHz}$.

\section{Observation}

Of the available CNA measurements, we extracted data from 1996 to 1998 , for which corresponding optical measurements from the MSP were available. The data was then narrowed down to those for winter and non-overcast days, when optical observation of aurora was possible, with CNA and auroral emission of at least $0.1 \mathrm{~dB}$ and $0.1 \mathrm{kR}$. The final data set of 79 days was then put to comparative analysis. MSP observation data were used to eliminate data for overcast days.

Figure 1 shows the observation data obtained at 11:12 to 13:00 UT on 3 December 1997. Figure 1a, b and c show the CNA, 427.8-nm and 630.0-nm intensity for the direction of geomagnetic zenith, respectively. Figure $1 \mathrm{~d}$ is the keogram of 557.7-nm emission, while Fig. 1e indicates the $X$ (south-north) component of the geomagnetic field (baseline: $12510 \mathrm{nT}$ ). As shown in the keogram, the auroral arc appeared on the polar side at 11:25 UT, traveled across the geomagnetic zenith toward the equator. The 630.0-nm intensity, 427.8-nm intensity and CNA peaked at 11:36 to 11:42 UT and gradually decreased thereafter. During this period, the geomagnetic field showed moderate activity up to $400 \mathrm{nT}$ reduction.

In Fig. 2, the measurements of 427.8-nm and 630.0-nm intensity are used to obtain the total energy flux $Q_{0}$ and characteristic energy $E_{c}$, and the observed CNA values are scatter plotted against $E_{c}$. CNA is proportional to the square root of the ion pair production rate $q$ from Eqs. (2) and (3), while $q$ is proportional to the total energy flux of precipitating electrons $Q_{0}$. The ordinate is therefore normalized as CNA per unit total energy flux, as obtained through dividing CNA with the square root of $Q_{0}$. The solid line in the figure represents the estimated curve of CNA against $E_{c}$, according to the theoretical calculation assuming that the energy distribution of precipitation electrons is represented by a Maxwellian distribution. In this example, the scatter of CNA data is distributed along the estimated curve. The agreement of predicted and observed CNA on this day is, however, an exception.

Figure 3 is a more typical example, showing the observations from 5:00 to 16:00 UT on 10 February 1997, when active auroras were observed frequently for an extended period of time. The data are shown in the same format as Fig. 1. The baseline of the geomagnetic $\mathrm{X}$ (south-north) component in Fig. 3e is 13210 nT. CNA and the 427.8-nm intensity began to increase gradually at around 6:00 UT, and reached the peak at 12:00 UT, when the geomagnetic field recorded the minimum of $-1300 \mathrm{nT}$. CNA maintained roughly the same 

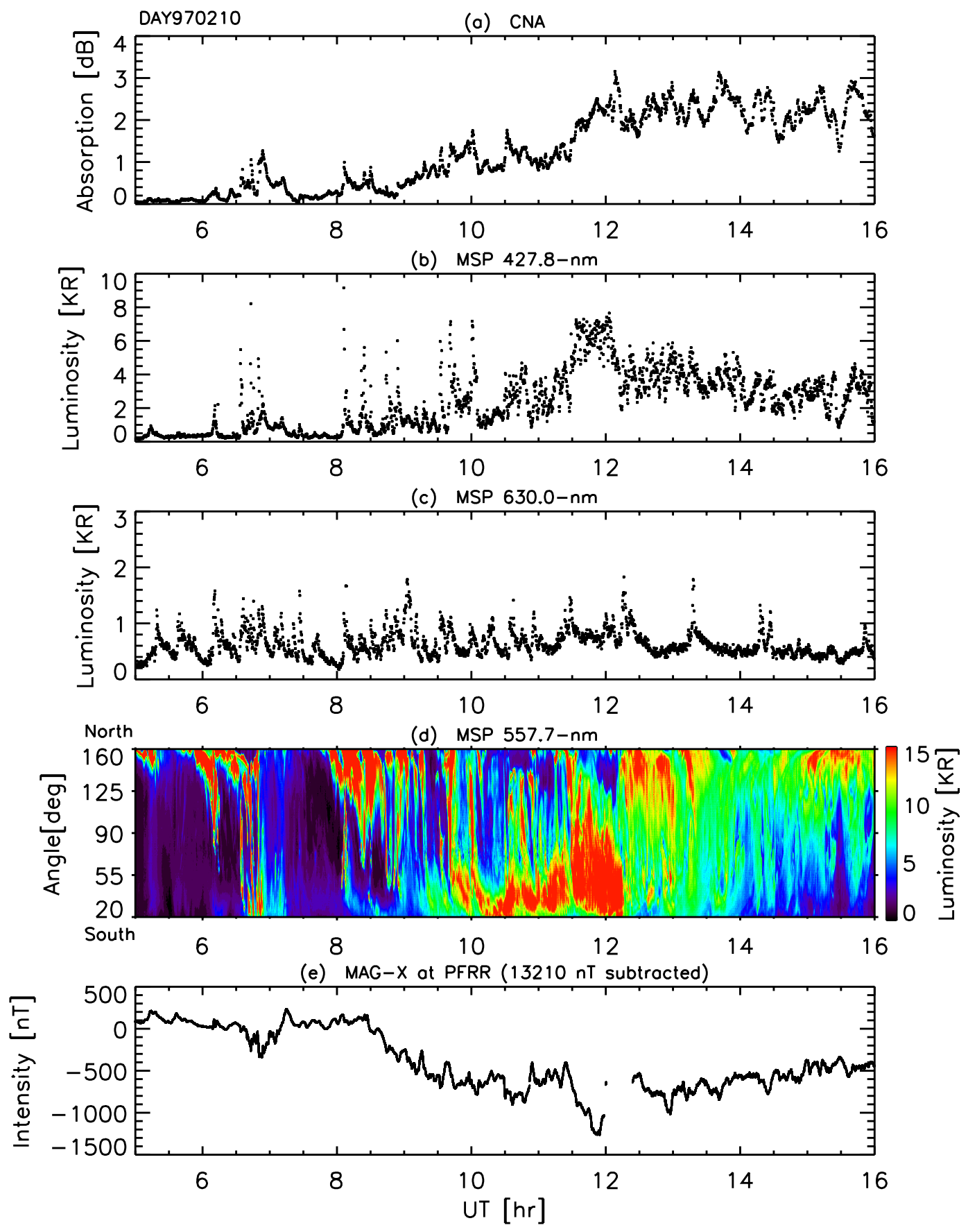

Fig. 3. Observed results for 5:00 to 16:00 UT on 10 February 1997, when active auroras were observed frequently. The format of this figure is the same as that of Fig. 1 .

level thereafter, but the 427.8-nm intensity showed a declining trend. The 630.0-nm intensity, on the other hand, showed frequent short fluctuations until 12:30 UT, indicative of soft electron precipitation, then the activities subsided thereafter.

Figure 4 is a scatter plot of CNA against $E_{c}$, according to the observation results shown in Fig. 3. It is in the same format as Fig. 2. Unlike the example of Fig. 2, this case shows the CNA derived points scattered over a wide area, significantly above the theoretically estimated curve (solid line).
This indicates that the observed CNA values are significantly larger than theoretically predicted using a Maxwellian distribution function.

In order to make a statistical comparison of the CNA measurements and the theoretical Maxwellian predicted values, a scatter plot was generated for all of the selected 79 days. Figure 5 shows the result of the comparison in the same format as Figs. 2 and 4. The solid line represents the theoretically estimated curve of CNA, as calculated on the assumption 


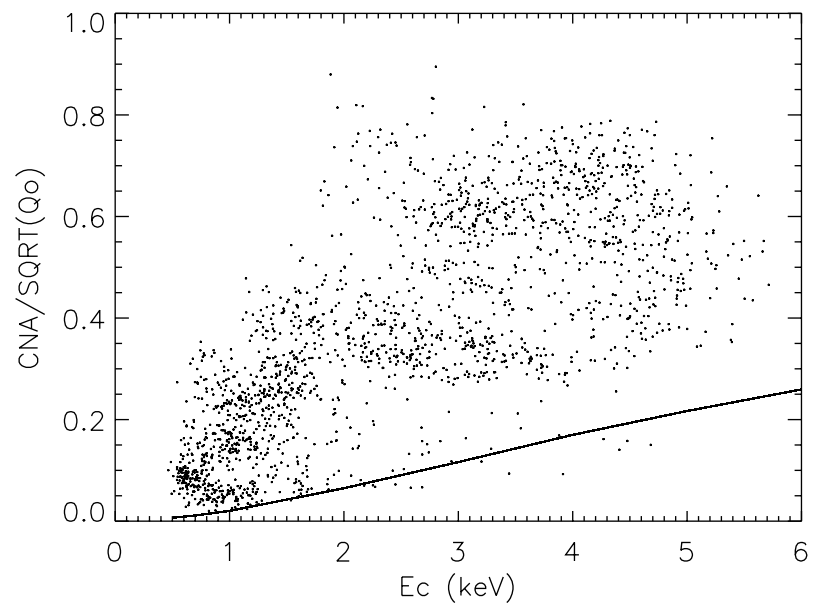

Fig. 4. Scatter plot of all CNA measurements shown in Fig. 3 against characteristic energy $E_{c}$. The format of this figure is the same as that of Fig. 2. Unlike the case of Fig. 2, the observed CNA is distributed significantly above the theoretically estimated curve (solid line).

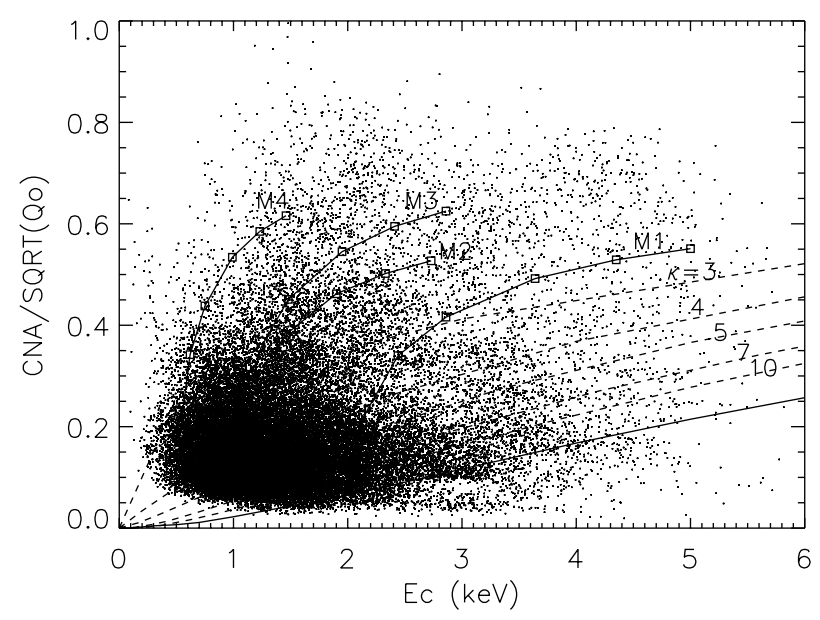

Fig. 5. Scatter plot of CNA against characteristic energy $E_{c}$ for all 79-day observations selected from the data set from 1996 to 1998. The format of this figure is the same as that of Figs. 2 and 4. The solid line in the figure represents the theoretically estimated curve with Maxwellian distribution assumed as the energy distribution of precipitating electrons. Most observations show a wide distribution significantly above the predicted curve. The five broken lines in the figure represent the theoretically estimated curve from kappa distributions ( $\kappa$ index of 3, 4, 5, 7 and 10) for the energy distribution of precipitating electrons. The solid lines with squares labeled M1, M2, M3, and M4 show the CNA calculated from double Maxwellian distributions. The parameters for the double Maxwellians are ( $E_{1}$ is the peak of the low energy, $E_{2}$ is the peak of the high energy Maxwellian) M1: $E_{1}=2 \mathrm{keV}, E_{2}=20 \mathrm{keV}$; M2: $E_{1}=1 \mathrm{keV}, E_{2}=20 \mathrm{keV} ;$ M3: $E_{1}=1 \mathrm{keV}, E_{2}=40 \mathrm{keV} ; \mathrm{M} 4$ : $E_{1}=0.5 \mathrm{keV}, E_{2}=40 \mathrm{keV}$. The ratio of the energy flux between the low and high energy Maxwellian was varied from 20 to 0.5 to give the plotted variation of the characteristic energy. that the energy distribution of precipitating electrons represents a Maxwellian distribution, and based on the MSIS-90 (f10.7=72, $\mathrm{A}_{p}=18$ ) atmospheric model and average atmospheric parameters. The broken lines and the solid lines labeled M1, M2, M3, and M4 will be discussed in the following section. There are no points around the origin of the coordinates, because CNA under $0.1 \mathrm{~dB}$ and optical data under $0.1 \mathrm{kR}$ are eliminated for lack of reliability. This figure shows that most CNA measurements are widely distributed significantly above the theoretical Maxwellian estimated curve. The result confirms that the example of Fig. 4 is a representative case.

\section{Discussion}

In order to identify the cause of the statistical difference between CNA measurements and its prediction based on optical observations, we first evaluate the uncertainty of the MSIS90 atmospheric model, effective recombination coefficient, specific absorption, and the relation between the brightness ratio and $E_{c}$, used in calculating the estimated values. Figure 6 shows the results of this evaluation. The solid line in Fig. 6a-d is the theoretically estimated curve based on the average parameters, the same as the solid line in Fig. 5. Figure 6a refers to the uncertainty caused by the MSIS-90 atmospheric model, which depends on the parameters of f10.7 and $\mathrm{A}_{p}$ index. These parameters varied between 66 to 121 and 0 to 59 , respectively, during the period of the 79-day data set. The two broken lines in the panel illustrate the range of variations for the theoretically estimated CNA curve, based on these variations of the f10.7 and $A_{p}$ index in the MSIS- 90 atmospheric model.

The next evaluation concerns the uncertainties in the effective recombination coefficient. Although the coefficient will not change much in the E-region, a significant change is theoretically predicted in the D-region due to factors such as seasons, day/night, ionization rate, etc. (e.g. Torkar and Friedrich, 1983; Velinov et al., 1984; Kirkwood and Osepian, 1995). In our case, CNA data are limited to those taken on winter nights in the polar region. Collis et al. (1984) compared the energetic electron data, measured by a geostationary satellite during auroral activities, and CNA measurements at the geomagnetically conjugate point, to obtain the average altitude distribution of the effective recombination coefficient at high latitude during auroral absorption events (Collis et al., 1984, Fig. 12b). The altitude distribution of the effective recombination coefficient (Fig. 15 in Hargreaves and Devlin, 1990), which we used in predicting CNA, refers to this distribution. The effective recombination coefficient for our 79-day data set is likely distributed around this average altitude profile. In order to evaluate how the uncertainty of this coefficient affects the theoretically estimated CNA curve, we shifted the coefficient values by a factor of 2 above and below the average values, to see how the curve would change. The result is shown in the two broken lines in Fig. 6b. The broken lines run above and below the 
(a)

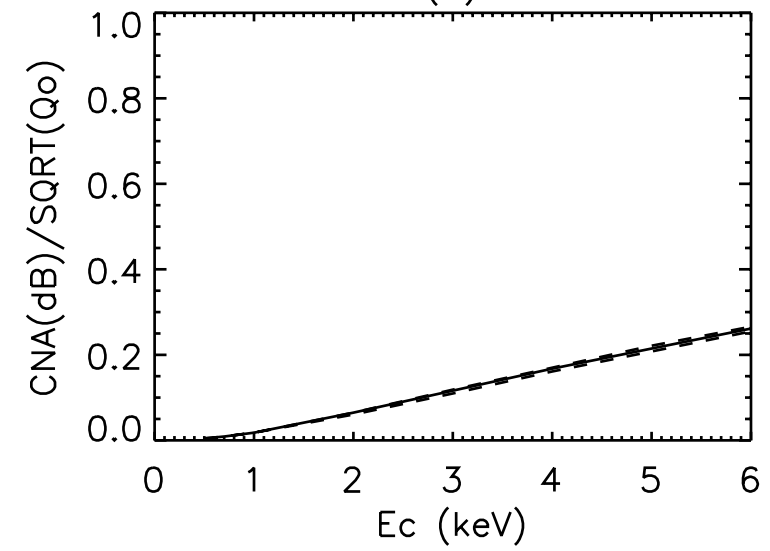

(c)

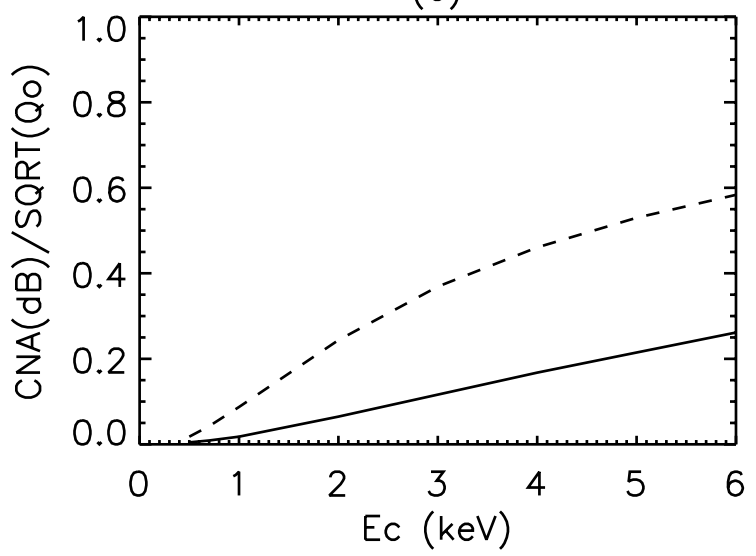

(b)

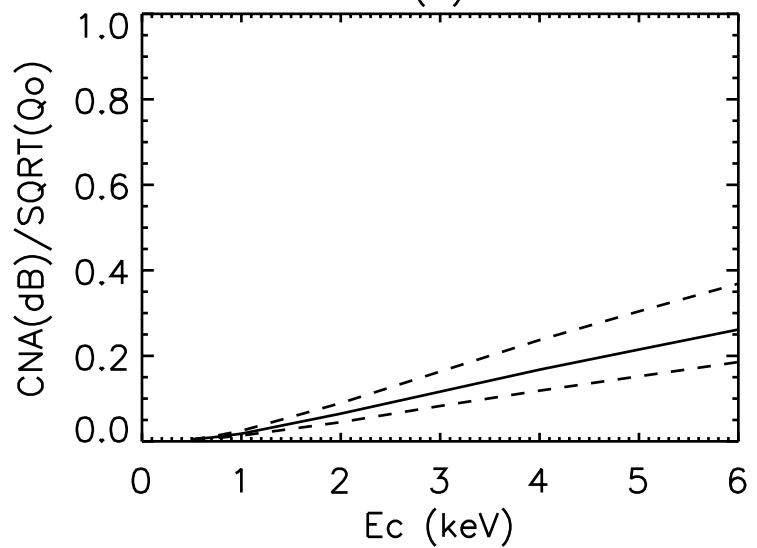

(d)

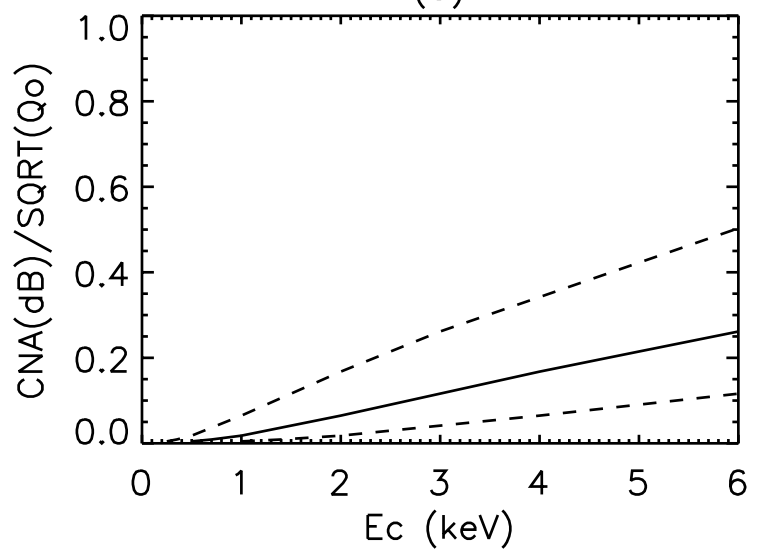

Fig. 6. Variation range of the theoretically estimated curve (broken lines), when the uncertainty of various atmospheric parameters are taken into consideration. The solid line in each figure represents the theoretically estimated curve using average atmospheric parameters. (a) Variation range corresponding to the variation of the parameters $\mathrm{f} 10.7$ and $\mathrm{A}_{p}$ index in the atmospheric model MSIS-90 during the period of 79-day data set. (b) Variation range corresponding to the increase and decrease of the effective recombination coefficient by a factor of 2. (c) Theoretically estimated curve when the electron temperature in the E-region of ionosphere rises to $2000^{\circ} \mathrm{K}$ (broken line). (d) Variation range corresponding to the increase and decrease by a factor of 2 of the conversion relation of the ratio of $427.8 \mathrm{~nm}$ and $630.0 \mathrm{~nm}$ brightness into the characteristic energy $E_{c}$.

theoretically estimated average curve (solid line) by a factor of $\sqrt{2}$. We then evaluate the uncertainty of the specific absorption $a(h)$. As shown in the Eq. (4), $a(h)$ is proportional to the electron-neutral collision frequency $v_{e n}$, which tends to increase as the electron temperature increases. However, since variations of electron temperature are small at the altitude below the D-region, $v_{\text {en }}$ also has only minor variations in this region (Aggarwal et al., 1979). Yet, in the polar region, geomagnetic activities can sometimes increase electron temperature in the E-region excessively. In such an event, the increase of $v_{e n}$ in the E-region is expected to increase CNA. According to the comparison of incoherent scatter radar and riometer measurements by Stauning (1984), when the electron temperature at the altitude of $110 \mathrm{~km}$ increased unusually and reached $2000^{\circ} \mathrm{K}$, a CNA of $\sim 0.6 \mathrm{~dB}$ was observed. The broken line in Fig. $6 \mathrm{c}$ is the theoretically estimated CNA curve, based on the $v_{e n}$ altitude profile, using the altitude distribution of electron temperature during its unusual increase in the E-region shown in Fig. 3 of Stauning (1984). The result shows that rising electron temperature in the E-region has the effect of raising the theoretically estimated curve. Finally, we evaluate the uncertainty of the $E_{c}$ calculation based on Fig. 7 by Lummerzheim et al. (1990), used to obtain $E_{c}$ from the brightness ratio of $\mathrm{I}(630.0) / \mathrm{I}(427.8)$. According to this figure, the average curve, shown in the solid line, has a standard deviation of around a factor of 2 . The two broken lines in Fig. 6d indicate the range of the theoretically estimated CNA curve, when this uncertainty is incorporated. These curves (broken lines) also present the range of a factor of 2 above and below the average curve (solid line). When the effects of uncertainties in the above four parameters are considered, the variation of the MSIS-90 atmospheric model, shown in Fig. 6a, has an extremely small effect. The uncertainty in the effective recombination coefficient, shown in Fig. 6b, and in the relationship between the intensity ratio and $E_{c}$, shown in Fig. 6d, both have the probability of 
shifting the theoretically estimated CNA curve above or below the average curve. Therefore, it should not be considered as the cause of actual measurements distributed systematically above the average curve. Although an abnormal enhancement in electron temperature in the E-region tends to shift the theoretically estimated curve upwards, as shown in Fig. 6c, such events are not likely to occur so often as to move the distribution of CNA measurements above the average curve in a statistically significant level. Consequently, we can eliminate the uncertainty of these four parameters as the cause for the statistically significant difference between the CNA measurements and theoretically estimated values.

For the interpretation of auroral brightness ratios, downward isotropic Maxwellian distributions are assumed as the energy distribution of precipitating electrons. The systematic underestimation of the CNA indicates that more high energy electrons are precipitating than contained in a Maxwellian spectrum. Since the source of precipitating electrons is in the plasma sheet region of the magnetotail, the shape of electron energy distribution in the plasma sheet should be reflected in the energy distribution of precipitating electrons. There are many satellite observation data available for electron energy distribution in the plasma sheet region (e.g. Vasyliunas, 1968; Christon et al., 1988, 1989, 1991). These observations show that the typical energy distribution $f(E)$ of the differential-directional electron number flux in this region is approximated by a kappa distribution which can be approximated with the formula:

$f(E)=A E\left(1+\frac{E}{\kappa E_{0}}\right)^{-\kappa-1}$.

Here, $E_{0}$ represents the energy at the maximum in the $f(E)$ function. Integrating Eq. (5) over directional angles and energy shows that the total energy flux $Q_{0}$ is related to the factor A as follows:

$Q_{0}=A \frac{2 \pi E_{0}^{3} \kappa^{2}}{(\kappa-1)(\kappa-2)}$.

In this distribution, the low-energy part $\left(E \leq \kappa E_{0}\right)$ has a Maxwellian form, whereas the high-energy tail $\left(E>>\kappa E_{0}\right)$ has a power-law form. The parameter $\kappa$ in the formula shows the gradient of the high energy power-law tail. The kappa distribution becomes the Maxwellian distribution as $\kappa$ reaches infinity (Vasyliunas, 1968). Therefore, instead of a Maxwellian distribution, a more general kappa distribution is assumed as the energy distribution of precipitating electrons, to calculate the theoretically estimated CNA curve. Other observations of high energy precipitating electrons suggest a double Maxwellian distribution (Codrescu et al., 1997). The additional high energy electrons have very little effect on the conversion of the brightness ratio to characteristic energy, as a re-evaluation of this ratio with the auroral model in Lummerzheim et al. (1990) shows. For the purpose of the auroral brightness ratio interpretation, Lummerzheim et al. (1990) use the term "characteristic energy" as defined by $E_{c}=\frac{1}{2}\langle E\rangle$, i.e. half of the mean energy, rather than the peak energy $E_{0}$. For the approximation to the kappa distribution shown in Eq. (5), the relation between $E_{c}$ and $E_{0}$ can be calculated analytically:

$E_{c}=\frac{E_{0} \kappa}{\kappa-2}$.

The broken lines in Fig. 5 are the theoretically estimated curves, based on kappa distributions with a $\kappa$ index of 3, 4, 5, 7 and 10. The constant $A$ is chosen with the condition that the energy flux of the distribution is the same as that of a Maxwellian distribution at characteristic energy $E_{c}$. The solid lines labeled M1, M2, M3, and M4 show the predicted CNA using double Maxwellian shaped spectra. These spectra are constructed from the sum of two Maxwellian distributions where lower energy Maxwellian has a peak energy at 2, 1,1 , and $0.5 \mathrm{keV}$ for M1, M2, M3, and M4, respectively, and the high energy Maxwellian has a peak energy of 20, 20, 40, and $40 \mathrm{keV}$, respectively. These values were chosen to cover a large range of energies and relative importance of the high energy tail. By varying the relative energy flux in these two Maxwellians from a ratio of 20 to 0.5 , the mean energy and thus the characteristic energy is varied over the range shown in the plot.

When these curves are compared against the scatter plot distribution, most observation results fall in the range between the theoretical predictions. Most points are within the range of the kappa distributions with $\kappa=3$ to $\infty$ (Maxwellian distribution) and the points above the kappa distributions can be explained with additional high energy precipitation, as simulated by the double Maxwellians. From observations of electrons in the plasma sheet, shown in Fig. 7 by Christon et al. (1989), the $\kappa$ index is typically in the range from 3 to 11 , with a most probable value between 5 and 6 . The comparison between this distribution and Fig. 5 indicates the same lowest values for the $\kappa$ index. While plasma sheet electrons are statistically of non-Maxwellian distribution, the scatter plot in Fig. 5 shows a broader distribution encompassing the area of Maxwellian distributions. This result may be attributable to thermalization of kappa distributed electrons in the plasma sheet in the process of magnetospheric transport or field-aligned precipitation. The findings indicate that the discrepancy between CNA measurements and the theoretical Maxwellian distribution estimated values can be explained well, if we assume that the energy distribution of precipitating electrons is not a simple Maxwellian distribution but has an additional high energy component, represented either by a second Maxwellian or by a kappa distribution which has a Maxwellian form in the low-energy part and a power-law form in the high-energy tail.

\section{Summary}

With the objective of estimating the energy distribution of precipitating electrons in the polar region, we conducted a statistical comparison between the CNA measurements, taken from the 256-element imaging riometer at Poker Flat, Alaska, and auroral observation data, taken from the MSP 
(Meridian Scanning Photometer). Observations in the direction of geomagnetic zenith taken during 79 days in the winter periods of 1996 to 1998 were used for the analysis.

According to the conventional method of using auroral emissions to estimate the energy of precipitating electrons, Maxwellian distributions were assumed as the electron energy distribution to process the measurements of auroral 427.8-nm and 630.0-nm, to obtain the total energy flux and characteristic energy. The results were combined with the average atmospheric model to estimate CNA and compare it against actual CNA measurements. The observed CNA had greater values than the theoretical prediction in a statistically significant scale.

To identify the cause, we evaluated the uncertainty in the CNA estimation caused by the MSIS-90 atmospheric model, effective recombination coefficient, specific absorption, and characteristic energy derivation model, as well as energy distribution of precipitating electrons. The results showed that uncertainty of atmospheric parameters could be eliminated as a major cause of the observed discrepancy. The distribution of actual measurements was better explained when CNA was estimated from energy distribution of precipitating electrons with double Maxwellian or kappa distributions. These represent typical energy distributions of electrons in the plasma sheet region of the magnetosphere. This finding suggests that the energy distribution of precipitating electrons reflects the shape of electron energy distribution in the plasma sheet.

Acknowledgements. We wish to express our deepest gratitude to R. Smith, Director of the Geophysical Institute of University of Alaska Fairbanks (GI/UAF), for supporting our cooperative study. We also would like to thank D. Rice (currently with the University of Utah) and technical staff of GI/UAF for assisting in operation of the imaging riometer.

Topical editor M. Lester thanks S. P. Christon and D. S. Evans for their help in evaluating this paper.

\section{References}

Aggarwal, K. M., Nath, N., and Setty, C. S. G. K.: Collision frequency and transport properties of electrons in the ionosphere, Planet. Space Sci., 27, 753-768, 1979.

Ansari, Z. A.: The aurorally associated absorption of cosmic noise at College, Alaska, J. Geophys. Res., 69, 4493-4513, 1964.

Butler, J. and Lowe, R.: Beam-forming matrix simplifies design of electronically scanned antennas, Electron. Des., 12, 170-173, 1961.

Callis, L. B.: Comment on "The diffuse aurora: A significant source of ionization in the middle atmosphere" by Frahm, R. A. et al., J. Geophys. Res., 105, 15 681-15 684, 2000.

Christon, S. P., Michell, D. G., Williams, D. J., Frank, L. A., Huang, C. Y., and Eastman, T. E.: Energy spectra of plasma sheet ions and electrons from $\sim 50 \mathrm{eV} / \mathrm{e}$ to $\sim 1 \mathrm{MeV}$ during plasma temperature transitions, J. Geophys. Res., 93, 2562-2572, 1988.

Christon, S. P., Williams, D. J., Michell, D. G., Frank, L. A., and Huang, C. Y.: Spectral characteristics of plasma ion and electron populations during undisturbed geomagnetic conditions, J. Geophys. Res., 94, 13 409-13 424, 1989.
Christon, S. P., Williams, D. J., Mitchell, D. G., Huang, C. Y., and Frank, L. A.: Spectral characteristics of plasma sheet ion and electron populations during disturbed geomagnetic conditions, J. Geophys. Res., 96, 1-22, 1991.

Codrescu, M. V., Fuller-Rowell, T. J., Roble, R. G., and Evans, D. S.: Medium energy particle precipitation influences on the mesosphere and lower thermosphere, J. Geophys. Res., 102, $19977-$ 19987, 1997.

Collis, P. N., Hargreaves, J. K., and Korth, A.: Auroral radio absorption as an indicator of magnetospheric electrons and of conditions in the disturbed auroral D-region, J. Atmos. Terr. Phys., 46, 21-38, 1984.

Detrick, D. L. and Rosenberg, T. J.: A phased-array radiowave imager for studies of cosmic noise absorption, Radio Sci., 25, 325338, 1990.

Frahm, R. A., Winningham, J. D., Sharber, J. R., Link, R., Crowley, G., Gaines, E. E., Chenette, D. L., Anderson, B. J., and Potemra, T. A.: The diffuse aurora: A significant source of ionization in the middle atmosphere, J. Geophys. Res., 102, 28 203-28 214, 1997.

Frahm, R. A., Winningham, J. D., Sharber, J. R., Link, R., Crowley, G., Gaines, E. E., Chenette, D. L., Anderson, B. J., and Potemra, T. A.: Reply, J. Geophys. Res., 105, 15 685-15 687, 2000.

Hargreaves, J. K. and Devlin, T.: Morning sector electron precipitation events observed by incoherent scatter radar, J. Atmos. Terr. Phys., 52, 193-203, 1990.

Hedin, A. E.: Extension of the MSIS thermosphere model into the middle and lower atmosphere, J. Geophys. Res., 96, 1159-1172, 1991.

Holt, O. and Omholt, A.: Auroral luminosity and absorption of cosmic radio noise, J. Atmos. Terr. Phys., 24, 467-474, 1962.

Jackman, C. H., Frederick, J. E., and Stolarski, R. S.: Production of odd nitrogen in the stratosphere and mesosphere: An intercomparison of source strengths, J. Geophys. Res., 85, 7495-7505, 1980.

Johansen, O. E.: Variations in energy spectrum of auroral electrons detected by simultaneous observation with photometer and riometer, Planet. Space Sci., 13, 225-235, 1965.

Kirkwood, S. and Osepian, A.: Quantitative studies of energetic particle precipitation using incoherent scatter radar, J. Geomag. Geoelectr., 47, 783-799, 1995.

Little, C. G. and Leinbach, H.: The riometer - a device for the continuous measurement of ionospheric absorption, Proc. IRE, 47, 315-320, 1959.

Lummerzheim, D., Rees, M. H., and Romick, G. J.: The application of spectroscopic studies of the aurora to thermospheric neutral composition, Planet. Space Sci., 38, 67-78, 1990.

Murayama, Y., Mori, H., Kainuma, S., Ishii, M., Nishimuta, I., Igarashi, K., Yamagishi, H., and Nishino, M.: Development of a high-resolution imaging riometer for the middle and upper atmosphere observation program at Poker Flat, Alaska, J. Atmos. Terr. Phys., 59, 925-937, 1997.

Parthasarathy, R. and Berkey, F. T.: Multiple-frequency investigations of radio wave absorption during the dawn-breakup phase of auroras, Radio Sci. J. Res. NBS/USNC-URSI, 69D, 415-421, 1965.

Rees, M. H.: Auroral ionization and excitation by incident energetic electrons, Planet. Space Sci., 11, 1209-1218, 1963.

Rees, M. H. and Luckey, D.: Auroral electron energy derived from ratio of spectroscopic emissions 1 . Model computations, J. Geophys. Res., 79, 5181-5185, 1974. 
Sharber, J. R., Frahm, R. A., Link, R., Crowley, G., Winningham, J. D., Gaines, E. E., Nightingale, R. W., Chenette, D. L., Anderson, B. J., and Gurgiolo, C. A.: UARS particle environment monitor observations during the November 1993 storm: Auroral morphology, spectral characterization, and energy deposition, J. Geophys. Res., 103, 26 307-26322, 1998.

Stauning, P.: Absorption of cosmic noise in the E-region during electron heating events, A new class of riometer absorption events, Geophys. Res. Lett., 11, 1184-1187, 1984.
Torkar, K. M. and Friedrich, M.: Tests of an ion-chemical model of the D- and lower E-region, J. Atmos. Terr. Phys., 45, 369-385, 1983.

Valentine, J. M. and Curran, S. C.: Average energy expenditure per ion pair in gasses and gas mixtures, Rep. Prog. Phys., 21, 1, 1958.

Vasyliunas, V. M.: A survey of low-energy electrons in the evening sector of the magnetosphere with OGO 1 and OGO 3, J. Geophys. Res., 73, 2839-2883, 1968.

Velinov, P., Smirnova, N. A., and Vlascov, V. A.: Hybrid quadriionic model of the lower ionosphere, Adv. Space Res., 4, 123130, 1984. 